\title{
HOTAIR, a long non-coding RNA driver of malignancy whose expression is activated by FOXC1, negatively regulates miRNA-1 in hepatocellular carcinoma
}

\author{
DONG-NA SU ${ }^{1}$, SHI-PIN WU ${ }^{1}$, HONG-TAO $\mathrm{CHEN}^{1}$ and JIN-HUA HE ${ }^{2}$ \\ ${ }^{1}$ Department of Infectious Diseases, Shenzhen People's Hospital, The Second Affiliated Hospital of Jinan University, Shenzhen, \\ Guangdong 518020; ${ }^{2}$ Department of Laboratory, Panyu Central Hospital, Guangzhou, Guangdong 511400, P.R. China
}

Received May 23, 2015; Accepted August 12, 2016

DOI: $10.3892 / \mathrm{ol} .2016 .5127$

\begin{abstract}
Evidence is rapidly accumulating that long non-coding RNAs (lncRNAs) are involved in human tumorigenesis and are dysregulated in multiple cancers, including hepatocellular carcinoma (HCC). IncRNAs can regulate essential pathways that contribute to tumor initiation and progression with tissue specificity, which suggests that lncRNAs may be valuable biomarkers and therapeutic targets. HOX transcript antisense intergenic RNA (HOTAIR) has previously been demonstrated to be an oncogene and a negative prognostic factor in a variety of cancers; however, the factors that contribute to the upregulation of HOTAIR and the interaction between HOTAIR and microRNAs (miRNAs or miRs) are largely unknown. In the present study, the expression levels of HOTAIR, forkhead box C1 (FOXC1) and miRNA-1 were examined in 50 matched pairs of HCC and HCC cells. The effects of HOTAIR on HCC cell proliferation were tested using trypan blue exclusion assay. The effect of HOTAIR on HCC growth in vivo was determined in a (nu/nu) mouse model. A computational screening of HOTAIR promoter was conducted to search for transcription factor-binding sites. FOXC1 binding to the promoter region of HOTAIR was confirmed using a chromatin immunoprecipitation assay. A search for miRNAs that had complementary base paring with HOTAIR was performed utilizing an online software program.
\end{abstract}

Correspondence to: Dr Shi-Pin Wu, Department of Infectious Diseases, Shenzhen People's Hospital, The Second Affiliated Hospital of Jinan University, 1017 Dong Men Bei Lu, Luo Hu, Shenzhen, Guangdong 518020, P.R. China

E-mail: sudona@qq.com

Abbreviations: lncRNA, long non-coding RNA; miR, microRNA; siRNA, small interfering RNA; TF, transcription factor; RT-qPCR, reverse transcription-quantitative polymerase chain reaction; $\mathrm{HCC}$, hepatocellular carcinoma; HOTAIR, HOX transcript antisense intergenic RNA; FOXC1, forkhead box C1

Key words: long non-coding RNA, HOTAIR, miR-1, FOXC1, hepatocellular carcinoma
The interaction between miR-1 and HOTAIR was examined using a luciferase reporter assay. Gain and loss of function approaches were used to determine the changes of HOTAIR or miR-1 expression. The relative levels of FOXC1 and HOTAIR expression in HCC tissues and HepG2 cells were significantly higher than those in normal liver LO2 cells and adjacent carcinoma tissues; the relative expression of miR-1 exhibited the opposite pattern. Overexpression of HOTAIR promoted HCC cell proliferation and progression of tumor xenografts. The present authors have demonstrated that FOXC1 binds to the upstream region of HOTAIR in HCC cells and that FOXC1 activates lncRNA HOTAIR expression in HCC HepG2 cells, which suggests that HOTAIR harbors a miRNA-1 binding site. The present data revealed that this binding site is vital for the regulation of miRNA-1 by HOTAIR. Furthermore, HOTAIR negatively regulated the expression of miRNA-1 in HepG2 cells. Additionally, the present study demonstrated that the oncogenic activity of HOTAIR is in part based on the negative regulation of miR-1. Taken together, these results suggest that HOTAIR is a FOXC1-activated driver of malignancy, which acts in part through the repression of miR-1.

\section{Introduction}

Hepatocellular carcinoma (HCC) is the third leading cause of cancer-associated mortalities, with nearly 600,000 mortalities occurring worldwide each year (1). Although resection is considered a potentially curative treatment for HCC patients, the 1-year post-operative survival rate is only $30-40 \%$ (2). Thus, it is necessary to improve our understanding of the disease-causing mechanisms and to identify specific biomarkers for HCC progression to aid in the prediction and improvement in clinical outcomes.

Non-coding RNAs (ncRNAs) are subdivided into two groups: Small ncRNAs (<200 nt) and long ncRNAs (lncRNAs) (3,4).SmallncRNAs, including the well-documented microRNAs (miRNAs or miRs), have received great attention, since they are important in cancer (5). It has been proposed that lncRNAs are involved in the epigenetic regulation of coding genes, and thus, exert a powerful effect on a number of physiological and pathological processes, including the pathogenesis of numerous human cancers (6). It has been reported that 
lncRNA HOX transcript antisense intergenic RNA (HOTAIR) expression is significantly higher in HCC tissues than in adjacent non-cancerous tissues (7). Patients with HCC tumors that overexpress HOTAIR have an increased risk of HCC recurrence following hepatectomy, and there is also a correlation between HOTAIR overexpression and increased risk of lymph node metastasis (8). The overexpression of HOTAIR is an independent prognostic factor for $\mathrm{HCC}$ recurrence in liver transplant patients (9). Furthermore, patients with high tumor expression of HOTAIR have a significantly shorter recurrence-free survival than patients with low expression of HOTAIR (10).

The mechanism by which HOTAIR exerts its oncogenic activity remains largely unknown. A regulatory mechanism has been proposed in which RNAs cross-talk via competing shared miRs (11). In addition, IncRNAs directly interact with RNA-binding proteins and localize to the gene promoter region to regulate gene expression (12). The proposed competitive endogenous RNAs (ceRNAs) mediate the bioavailability of miRs on their targets, thus imposing another level of post-transcriptional regulation (13). An example of this type of regulation is the IncRNA colon cancer associated transcript 1, (CCAT1), which binds miR-218-5p and forms a regulatory interaction (14). Using jaspar bioinformatics software (http://jaspar.genereg. net), forkhead box $\mathrm{C} 1$ (FOXC1) shared binding sites with the upstream promoter region of HOTAIR were predicted. Furthermore, miR-1 was identified as the miR that shares binding sites with HOTAIR using starBase 2.0 (http://starbase.sysu.edu.cn). Thus, the present authors hypothesized that transcription factors (TFs) activate the transcription of HOTAIR. Furthermore, HOTAIR overexpression inhibits the expression of miR-1. This hypothesis is discussed to better elucidate the pathogenesis of HCC.

\section{Materials and methods}

Tissue specimens. Fresh-frozen and paraffin-embedded HCC tissues and corresponding adjacent non-tumorous gastric samples were obtained from Chinese patients at Shenzhen People's Hospital (Shenzhen, China) between January 2010 and December 2014. All cases were reviewed by a pathologist and histologically confirmed as HCC. Informed consent was obtained from all patients and the study was approved by the Institutional Ethics Committee of Shenzhen People's Hospital.

Construction of recombinant HOTAIR lentiviral expression vectors. The sequence of HOTAIR was obtained (https:// www.ncbi.nlm.nih.gov/nuccore/383286742? report=fasta) and synthesized. Upon double digestion with BamHI and EcoRI, HOTAIR was directionally-connected to the pGLV3/H1/GFP/ Puro vector and then transformed into DH5 $\alpha$ competent cells (Shanghai GenePharma Co., Ltd., Shanghai, China). Recombinant, packaging and envelope plasmids (Shanghai GenePharma Co., Ltd.) were co-transfected into 293T cells for $72 \mathrm{~h}$. Viruses were then collected and the titers were determined using the dilution hole measurement method (15). The constructed recombinant HOTAIR lentiviral expression vector was designated LV3-HOTAIR (16).

Design and construction of a eukaryotic expression vector for Homo sapiens (hsa)-miR-1 and FOXC1. The mature hsa-miR-1 sequence (5'-UGGAAUGUAAAGAAGUAUGUAU-3') is available from the miR registry (MIMAT000416; http://www. mirbase.org/cgi-bin/mirna_entry.pl?acc=MIMAT0000416). To prevent the formation of a termination signal, TTGGCCACT GACT was selected as the region in a miR expression vector template (pcDNA3.1; Invitrogen; Thermo Fisher Scientific, Inc., Waltham, MA, USA). The sequence TGCT was added to the 5 ' sense strand template of the miR expression vector, while GTCC was added to the 5 ' antisense strand template (17). In addition, a non-specific sequence was designed and synthesized by Shanghai GenePharma Co., Ltd. The eukaryotic expression vector plasmid targeting hsa-miR-1 was designated pmiR-1. The sequence of FOXC1 was synthesized and sub-cloned into pcDNA3.1 (Invitrogen; Thermo Fisher Scientific, Inc.) to generate $\mathrm{pFOXC1.Empty} \mathrm{pcDNA3.1} \mathrm{vector} \mathrm{was} \mathrm{used} \mathrm{as} \mathrm{a} \mathrm{nega-}$ tive control (NC).

Cell culture. HCC HepG2 and LO2 cells were purchased from the Shanghai Institute of Cell Biology (Shanghai, China). The cell lines were cultured in Dulbecco's modified Eagle's medium (Gibco; Thermo Fisher Scientific, Inc.) containing 10\% fetal bovine serum (HyClone; GE Healthcare Life Sciences, Logan, UT, USA), $100 \mathrm{U} / \mathrm{ml}$ penicillin and $100 \mu \mathrm{g} / \mathrm{ml}$ streptomycin (Invitrogen; Thermo Fisher Scientific, Inc.). Cells were maintained in a humidified incubator at $37^{\circ} \mathrm{C}$ in the presence of $5 \%$ $\mathrm{CO}_{2}$. All cell lines were passaged for $<6$ months.

Cell transfection. All plasmid vectors (pmiR-1, $\mathrm{pFOXC1}$ and empty vector) used for cell transfection were extracted using Hieff Trans ${ }^{\mathrm{TM}}$ Liposomal Transfection Reagent (Yeasen Biotechnology Co., Ltd., Shanghai, China). HepG2 cells cultured on 6-well plates were transfected with pmiR-1, pFOXC1 or empty vector using Lipofectamine 2000 (Invitrogen; Thermo Fisher Scientific, Inc.) according to the manufacturer's protocol. Cells were harvested after $48 \mathrm{~h}$ for quantitative polymerase chain reaction (qPCR). Methods for transfecting cells with LV3-HOTAIR were adopted, as previously described (17). Small interfering RNAs (siRNAs) were designed and synthesized by Shanghai GenePharma Co., Ltd. The siRNA sequence used in the present study was as follows: Sense, 5'-GGGAGATGTTCGAGT CACAGA-3' and antisense, 5'-GCGTAAAGCTCGGGTAAG TAG-3'. The siRNAs sequences targeting human HOTAIR were as follows: Sense, 5'-GGAGAACACUUAAAUAAGUTT-3' and antisense, 5'-ACUUAUUUAAGUGUUCUCCTA-3' (18).

Trypan blue exclusion assay. The density of the HepG2 cell line suspension was determined by counting on a hemacytometer. A $0.4 \%$ solution of trypan blue in phosphate-buffered saline (PBS) was prepared ( $\mathrm{pH}$ 7.2-7.3). Trypan blue stock solution (0.1 ml) was added to $1 \mathrm{ml}$ of cells. A hemacytometer was loaded and examined immediately under a microscope at low magnification. The number of blue-stained cells and the number of total cells were counted. Cell viability was considered to be $\geq 95 \%$ for healthy log-phase cultures. The percentage of viable cells was calculated as follows: Viable cells $(\%)=[1.00-($ number of blue-stained cells/number of total cells)] x 100.

In vivo treatment. A total of $15 \mathrm{BALB} / \mathrm{c}(\mathrm{nu} / \mathrm{nu})$ male mice $(200 \pm 2.6 \mathrm{~g}$; age, 3 months) from the Animal Center of Guangzhou Province (Guangdong, China) received 
Table I. Primers for quantitative polymerase chain reaction.

\begin{tabular}{ll}
\hline Gene & \multicolumn{1}{c}{ Primers $\left(5^{\prime}-3^{\prime}\right)$} \\
\hline U6 & F: CTCGCTTCGGCAGCACA \\
U6 & R: AACGCTTCACGAATTTGCGT \\
$18 S$ & F: CCTGGATACCGCAGCTAGGA \\
$18 S$ & R: AACGCTTCACGAATTTGCGT \\
HOTAIR & F: CAGTGGGGAACTCTGACTCG \\
HOTAIR & R: GTGCCTGGTGCTCTCTTACC \\
FOXC1 & F: CTCAACGAGTGCTTCGTCAA \\
FOXC1 & R: ACATGTTGTAGGAGTCCGGG \\
miR-1 & F: CACTCCAGCTGGGTGGAAT \\
& GTAAAGAAGTAT
\end{tabular}

The level of expression was calculated using $\mathrm{Cq}$ and the $2^{-\Delta \Delta \mathrm{Cq}}$ method (16). F, forward; R, reverse; HOTAIR, HOX transcript antisense intergenic RNA; FOXC1, forkhead box $\mathrm{C} 1$; miR, microRNA.
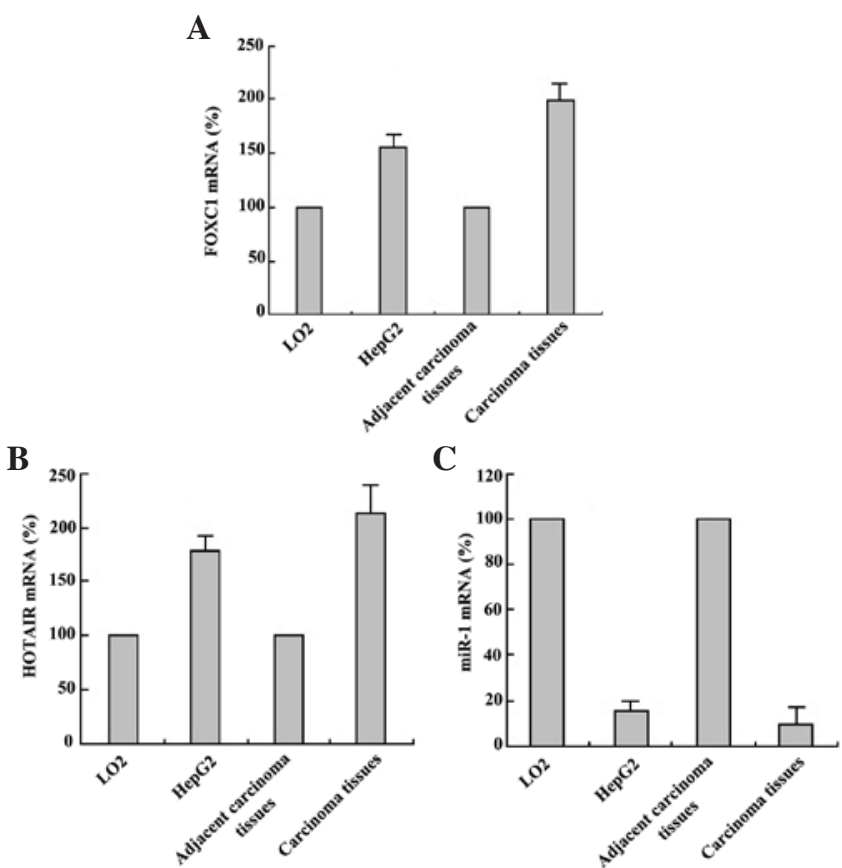

Figure 1. Relative levels of gene expression detected by reverse transcriptionquantitative polymerase chain reaction. Relative expression of (A) FOXC1, (B) miR-1 and (C) HOTAIR. HOTAIR, HOX transcript antisense intergenic RNA; FOXC1, forkhead box C1; miR, microRNA; mRNA, messenger RNA.

subcutaneous injections of $2 \times 10^{6} \mathrm{HepG} 2$ cells into the axillae bilaterally. The mice were housed in a pathogen-free environment at a temperature of $20-26^{\circ} \mathrm{C}$ and were exposed to $12 \mathrm{~h}$ light/dark cycles with free access to food and water. When xenograft tumors became palpable $\left(\sim 0.1 \mathrm{~mm}^{3}\right)$, mice were randomly divided ( $\mathrm{n}=5 \mathrm{mice} / \mathrm{group})$ into a control group receiving a PBS injection $(100 \mu 1)$, a transfection group receiving LV3-HOTAIR (200 $\mathrm{nM})$ and a NC group receiving $\mathrm{LV} 3$ + scramble sequence $(200 \mathrm{nM})$. There was no difference in the baseline tumor size between the groups. Tumor volume was calculated every 3 days according to the following formula: $V=a b 2 \pi / 6$, where ' $a$ ' is the maximum

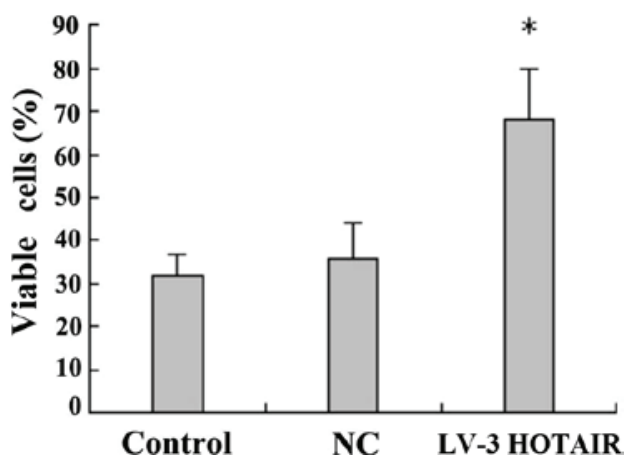

Figure 2. The proliferation of HepG2 cells was determined with trypan blue exclusion assay. Data are presented as the mean \pm standard deviation; $n=3$; ${ }^{*} \mathrm{P}<0.05$ vs. NC group. HOTAIR, HOX transcript antisense intergenic RNA; $\mathrm{NC}$, negative control.

tumor diameter and ' $b$ ' is the minimum tumor diameter. After treatment for 20 days, the mice were euthanized, and the tumors were extirpated and weighed.

Chromatin immunoprecipitation (ChIP) assay. HepG2 cells were treated with formaldehyde and incubated for $10 \mathrm{~min}$ to generate DNA-protein cross-links. Cell lysates were then sonicated to generate chromatin fragments of 200-300 bp and immunoprecipitated with anti-FOXC1 (cat. no. 625905; 1:1,000; R\&D Systems China Co., Ltd., Shanghai, China) for $1 \mathrm{~h}$ at room temperature or Alexa Fluor ${ }^{\circledR} 488$ conjugated-immunoglobulin $\mathrm{G}(\mathrm{IgG})$ antibody (cat. no. 4408; 1:1,000; Cell Signaling Technology, Inc., Danvers, MA, USA) for $30 \mathrm{~min}$ at room temperature as a control. Precipitated chromatin DNA was recovered and analyzed by qPCR.

Reverse transcription (RT)-qPCR. Total RNA samples were extracted using TRIzol (Invitrogen; Thermo Fisher Scientific, Inc.) according to the manufacturer's protocol. RT-qPCR analysis was performed using the Ultra SYBR Mixture with ROX (CWBio, Co., Ltd., Beijing, China) and an Applied Biosystems 7500 Real-Time PCR System (Thermo Fisher Scientific, Inc.). HOTAIR cDNA amplification was performed under the following conditions: Initial denaturation at $95^{\circ} \mathrm{C}$ for 5 min followed by 30 cycles of denaturation at $95^{\circ} \mathrm{C}$ for $30 \mathrm{sec}$ and primer annealing at $55^{\circ} \mathrm{C}$ for $30 \mathrm{sec}$, with a final extension step at $72^{\circ} \mathrm{C}$ for $60 \mathrm{sec}$. FOXC1 cDNA amplification was performed under the following conditions: Initial denaturation at $95^{\circ} \mathrm{C}$ for 5 min followed by 30 cycles of denaturation at $95^{\circ} \mathrm{C}$ for $30 \mathrm{sec}$ and primer annealing at $55^{\circ} \mathrm{C}$ for $30 \mathrm{sec}$, with a final extension step at $72^{\circ} \mathrm{C}$ for $60 \mathrm{sec}$. miR-1 cDNA was amplified under the following conditions: Initial denaturation at $95^{\circ} \mathrm{C}$ for $10 \mathrm{~min}$ followed by 40 cycles of denaturation at $95^{\circ} \mathrm{C}$ for $10 \mathrm{sec}$ and primer annealing at $57^{\circ} \mathrm{C}$ for $20 \mathrm{sec}$, with a final extension step at $72^{\circ} \mathrm{C}$ for $10 \mathrm{sec}$. The level of $18 \mathrm{~S}$ expression was used as an internal control for messenger RNAs, while the U6 level was used as an internal control for miRs. The primers used in RT-qPCR are indicated in Table I. The expression levels were calculated using the $2^{-\Delta \Delta \mathrm{Cq}}$ method (19).

Luciferase reporter assay. The full sequence HOTAIR gene was obtained by qPCR amplification using the Ultra 
A

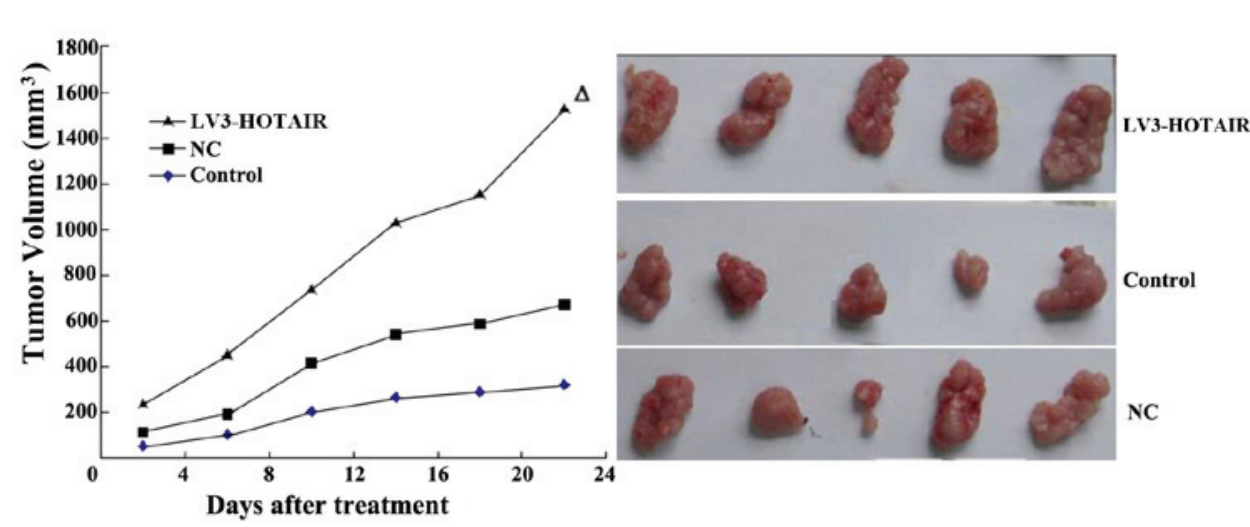

Figure 3. Effect of LV3-HOTAIR on hepatocellular carcinoma xenografts. HepG2 tumor xenografts were established in male athymic nude mice. Animals in the treatment group received LV3-HOTAIR $(200 \mathrm{nM} / \mathrm{kg}$ once daily). Control mice received phosphate-buffered saline (100 $\mu \mathrm{l} / \mathrm{kg}$ once daily). NC mice received scrambled sequence $(200 \mathrm{nM} / \mathrm{kg}$ once daily). After 20 daily treatments, tumors injected with LV3-HOTAIR were significantly larger than those in the control and NC groups. (A) Growth curves of tumor xenografts, ${ }^{\wedge} \mathrm{P}<0.05$ compared with control and NC groups. (B) Mice were sacrificed and their xenografts were removed for weighing. HOTAIR, HOX transcript antisense intergenic RNA; NC, negative control.

SYBR Mixture with ROX and an Applied Biosystems 7500 Real-Time PCR System. HOTAIR cDNA was amplified under the following conditions: Initial denaturation at $95^{\circ} \mathrm{C}$ for $5 \mathrm{~min}$ followed by 30 cycles of denaturation at $95^{\circ} \mathrm{C}$ for $30 \mathrm{sec}$ and primer annealing at $55^{\circ} \mathrm{C}$ for $30 \mathrm{sec}$, with a final extension step at $72^{\circ} \mathrm{C}$ for $60 \mathrm{sec}$. The gene was cloned separately into the multiple cloning site of the psi-CHECK ${ }^{\mathrm{TM}}-2$ luciferase miR expression reporter vector. HepG2 cells were transfected with miR mimic, miR inhibitor, control miR, negative control (NC), negative control inhibitor (all purchased from Guangzhou Leader Bio-Technology Co., Ltd., Guangzhou, China) or empty plasmid using Lipofectamine 2000, according to the manufacturer's protocol. Nucleotide-substitution mutation analysis was conducted using direct oligomer synthesis of full sequences. All constructs were verified by sequencing. Luciferase activity was measured using the Dual Luciferase Reporter Assay System Kit (Promega Corporation, Madison, WI, USA) on an Infinite M200 luminescence reader (Tecan Group Ltd., Männedorf, Switzerland), according to the manufacturer's protocol.

Data analysis. All results are the average of $\geq 3$ independent experiments from separately treated and transfected cultures. Data are expressed as the mean \pm standard deviation. Statistical comparisons were performed by one-way analysis of variance. $\mathrm{P}<0.05$ was considered to indicate a statistically significant difference.

\section{Results}

High expression of HOTAIR in HCC tissues and cells. HCC HepG2 and LO2 cells were cultured conventionally. Total RNA was extracted from tissues and cells using TRIzol. The relative expression levels of FOXC1, HOTAIR and miR-1 were detected by RT-qPCR. The relative level of FOXC1 and HOTAIR expression in HCC tissues and HepG2 cells was significantly higher than that in normal liver LO2 cells and adjacent carcinoma tissues (Fig. 1A and B). The relative expression of miR-1 exhibited the opposite pattern (Fig. 1C). These results indicate that HOTAIR has a tumor-promoting role in HCC, while miR-1 has a tumor-suppressor role, and there may be negative regulation at the level of expression between HOTAIR and miR-1. The high expression of FOXC1 may activate the transcription of HOTAIR. These results provided a foundation to further explore the mechanism of action of the HOTAIR transcript.

LV3-HOTAIR promotes HepG2 cell proliferation. Following Lipofectamine 2000 transfection of HepG2 cells with the lentiviral expression vector LV3-HOTAIR, cells were stained $72 \mathrm{~h}$ later with $0.04 \%$ trypan blue, and the cell survival rate was calculated. In the experimental group (LV3-HOTAIR), the effects on cell proliferation activity decreased significantly compared with those in the blank control and $\mathrm{NC}$ groups $(\mathrm{P}=0.01)$, indicating that LV3-HOTAIR can promote the proliferation of HepG2 cells (Fig. 2).

LV3-HOTAIR promotes the progression of tumor xenografts. HepG2 tumor xenografts were established in athymic nude mice to evaluate the effects of LV3-HOTAIR on HCC growth in vivo. Compared with the untreated animals, application of LV3-HOTAIR significantly diminished the tumor volume, whereas no effect was observed in the NC group (Fig. 3). No body weight loss or diarrhea was observed, and all animals (treated and non-treated) survived. The results demonstrated that the overexpression of HOTAIR can effectively inhibit HCC cancer growth in vivo.

FOXCl binds to the promoter region of HOTAIR and activates IncRNA HOTAIR expression. Recently, numerous important TFs have been demonstrated to be involved in regulating lncRNA transcription (20). To determine which TFs activate DD3 expression, the potential TF binding sites in the promoter region of HOTAIR were analyzed using the jaspar database (http://jaspar.genereg.net). The results revealed that there is one E-box element recognized by FOXC1. To further demonstrate that FOXC1 can directly bind to the HOTAIR promoter regions and activate the expression 


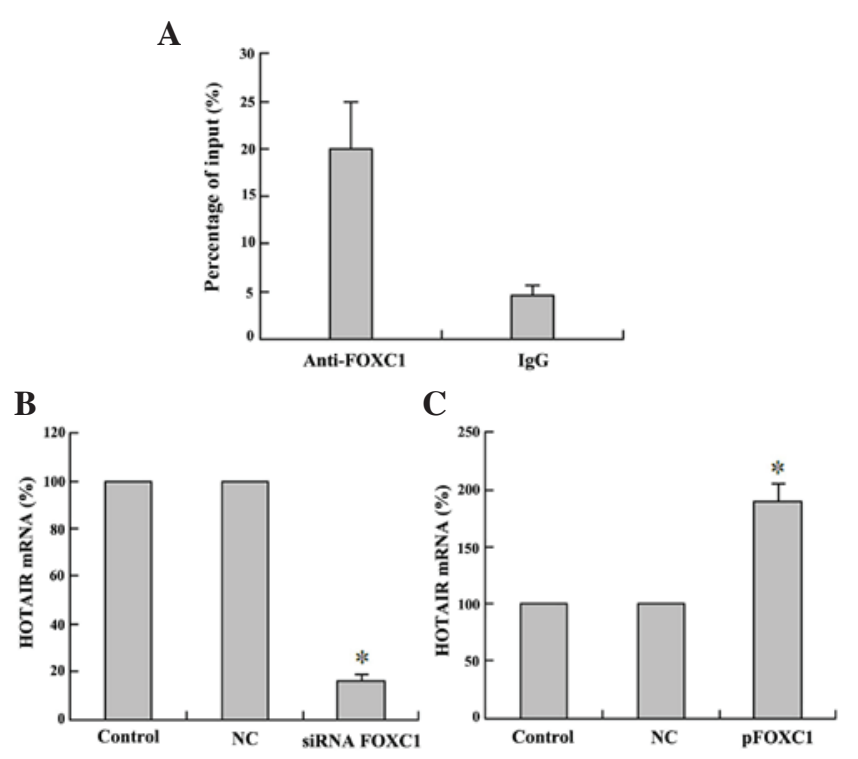

Figure 4. FOXC1 promotes HOTAIR expression via binding to its promoter regions. (A) ChIP assays were used to assess FOXC1 binding at the promoter region of HOTAIR containing the E-box element. The ChIP-derived DNA was amplified by quantitative polymerase chain reaction with specific primers and expressed as a percentage of input DNA. Data are presented as the mean \pm standard error based on $\geq 3$ independent experiments $($ $P<0.05$ compared with the IgG group). (B) Levels of HOTAIR expression following the transfection of siRNA FOXC1 or scrambled siRNA into HepG2 cells ("P $<0.05$ compared with the NC group). (C) Levels of HOTAIR upon transfection of pFOXC1 or empty vector into HepG2 cells ( $\mathrm{P}<0.05$ compared with the NC group). FOXC1, forkhead box C1; IgG, immunoglobulin G; ChIP, chromatin immunoprecipitation; HOTAIR, HOX transcript antisense intergenic RNA; NC, negative control; mRNA, messenger RNA; siRNA, small interfering RNA; p, plasmid.

of HOTAIR, FOXC1 immunoprecipitates were observed to be highly enriched in DNA fragments compared with the NC IgG immunoprecipitates in a ChIP assay. The ChIP assays indicated that FOXC1 directly bound to HOTAIR promoter regions (Fig. 4A). These results suggest that HOTAIR interacts with the FOXC1 responsive element in the HOTAIR promoter to induce transcription. Furthermore, the qPCR results indicated that HOTAIR expression was increased upon pFOXC1 transfection (Fig. 4B), while HOTAIR expression was decreased following siRNA-FOXC1 transfection (Fig. 4C). These results indicated that there is a positive regulation between the TF FOXC1 and the IncRNA HOTAIR. Taken together, these data suggest that the TF FOXC1 activates the expression of the IncRNA HOTAIR in HCC HepG2 cells.

HOTAIR negatively regulates the expression of miRNA-1. The function of lncRNAs in human diseases may reflect their ability to regulate gene expression. Increasing evidence has suggested that ncRNAs may participate in the ceRNA regulatory network (21). For example, there is a negative correlation between CCAT1 and let-7 (22). To confirm the direct binding between HOTAIR and miR-1, luciferase reporter constructs were generated (Fig. 5A). As predicted, there was one putative binding site in HOTAIR (1,615-1,638 bp). It was observed that co-transfection of HepG2 cells with miR-1 mimics and wild type psi-CHECK ${ }^{\mathrm{TM}}$-2-HOTAIR significantly inhibited luciferase activity $(\mathrm{P}=0.01$; Fig. $5 \mathrm{~B})$; however,
A

miR-1 3' 'TATGTATGAAGAAATGTAAGGT- 5'

| | | | | ।

HOTAIR 5'-AGACTCAGG ACTGCACAT TCCT- 3'
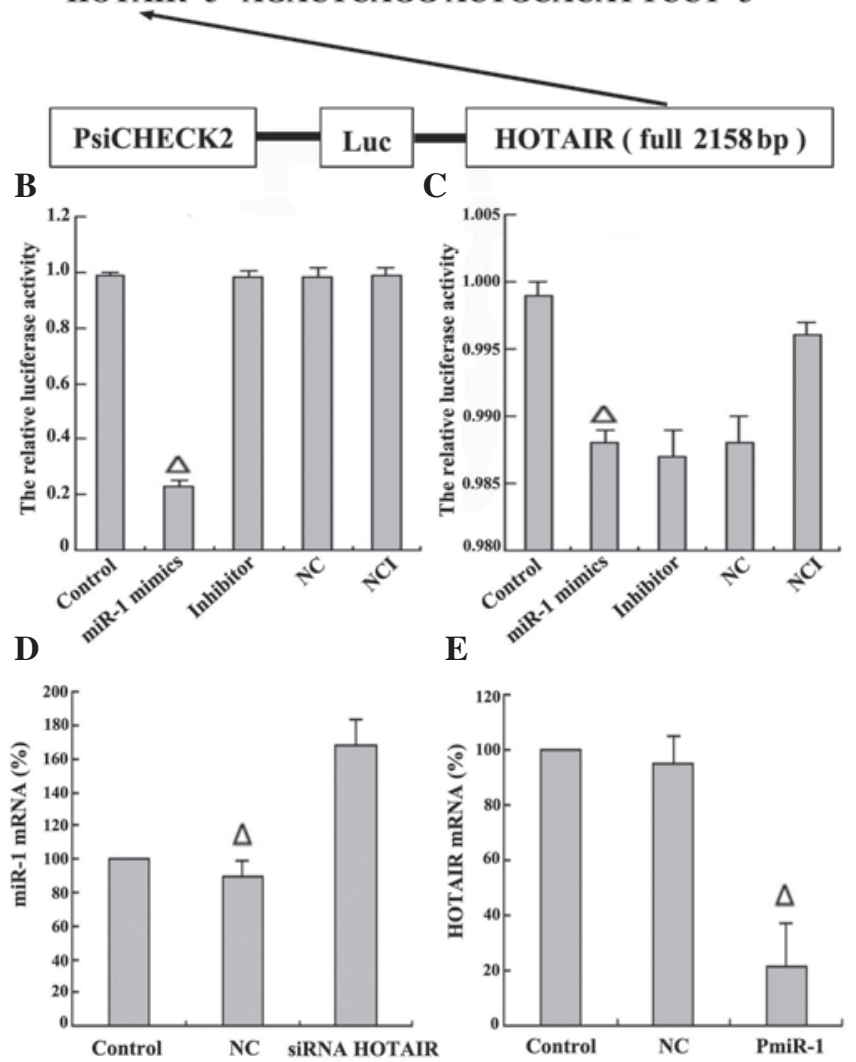

Figure 5. HOTAIR negatively regulates the expression of miR-1 in HepG2 cells. (A) Luciferase reporter plasmid containing the HOTAIR wild type sequence or a mutant transcript. (B) Comparison of luciferase activity of plasmid-transfected cloned HOTAIR full-length transcript $\left({ }^{\Delta} \mathrm{P}<0.05\right.$ vs. control and $\mathrm{NC}$ groups). (C) Comparison of the luciferase activity of the plasmid-transfected cloned HOTAIR mutant transcript ( $\mathrm{P}>0.05$ vs. control and $\mathrm{NC}$ groups). (D) HepG2 cells were treated with siRNA HOTAIR for $48 \mathrm{~h}$ and the relative levels of miR-1 expression were analyzed by RT-qPCR $\left({ }^{\Delta} \mathrm{P}<0.05\right.$ vs. control and $\mathrm{NC}$ groups). (E) HepG2 cells were treated with pmiR-1 for $48 \mathrm{~h}$ and the relative levels of HOTAIR expression were analyzed by RT-qPCR $\left({ }^{\Delta} \mathrm{P}<0.05\right.$ vs. control and NC groups). miR, microRNA; HOTAIR, HOX transcript antisense intergenic RNA; Luc, luciferase; NC, negative control; NCI, negative control inhibitor; mRNA, messenger RNA; siRNA, small interfering RNA; p, plasmid; RT-qPCR, reverse transcription-quantitative polymerase chain reaction.

co-transfection of HepG2 cells with miR-1 mimics and mutant psi-CHECK ${ }^{\mathrm{TM}}$-2-HOTAIR had little effect on the activity of luciferase ( $\mathrm{P}=0.09$; Fig. 5C). These data confirmed that miR-1 regulates the expression of HOTAIR by directly binding to target sites within the HOTAIR sequence. To further explore the regulatory association between HOTAIR and miR-1, the siRNA sequencing for HOTAIR was designed and transfected into HepG2 cells for 48 h to knock down the expression of HOTAIR. It was observed that the levels of miR-1 expression were significantly upregulated in contrast to those in the blank control (non-transfected siRNA HOTAIR) and NC (transfected with random sequences) groups (Fig. 5D). The eukaryotic expression vector plasmid of miR-1 was further constructed and transfected into HepG2 cells for $48 \mathrm{~h}$ to induce overexpression of miR-1. Upon overexpression of miR-1, the levels of HOTAIR expression were significantly downregulated in contrast to those in the blank 
control (non-transfected miR-1) and NC (transfected with plasmid and random sequences) groups (Fig. 5E). Thus, there may be a regulatory effect between HOTAIR and miR-1. To summarize, HOTAIR negatively regulated the expression of miR-1 in HepG2 cells.

\section{Discussion}

Recent evidence has suggested that ncRNAs serve an important role in cancer pathogenesis and can provide a novel insight into the biology of cancer (23). Over the past decade, research involving miRs has dominated the field of ncRNA regulation (24); however, the role of lncRNAs in the tumorigenesis of HCC remains largely unknown. Understanding the precise molecular mechanism by which IncRNAs function would facilitate the development of IncRNA-directed diagnostics and therapeutics against cancer. The present study provided evidence that FOXC1, a TF, activates the expression of HOTAIR, and that HOTAIR exhibits oncogenic activities in part through the modulation of miR-1. Therefore, HOTAIR promotes the tumorigenesis of HCC.

HOTAIR IncRNA was introduced by Kornienko et al (24) as a spliced and polyadenylated RNA with 2,158 nt and six exons (25). This RNA arises from the transcription of the antisense strand of the HOXC gene, which is specifically situated between HOXC11 and HOXC12 on chromosome $12 \mathrm{q} 13.13$ (25). HOTAIR is an oncogenic factor and can be used as a prognostic biomarker in different cancer types, since HOTAIR plays a key role in the initiation and progression of different types of cancer, including cervical cancer and nasopharyngeal carcinoma (26). HOTAIR, a lncRNA initially identified in breast cancer, was demonstrated to be upregulated in a variety of carcinomas $(27,28)$. A large number of studies have focused on the biological role and association of HOTAIR with clinical prognosis $(9,29,30)$, yet the precise factors regulating its expression remain largely unknown. HOTAIR is transcriptionally regulated by estradiol in breast cancer, which is tumor-specific (31). In the current study, a putative binding site of FOXC1 in the promoter region of HOTAIR was predicted. In addition, miR-1 was observed to have a binding site on HOTAIR. RT-qPCR results revealed that the relative level of FOXC1 and HOTAIR expression in HCC tissues and HepG2 cells was significantly higher than that in normal liver LO2 cells and adjacent carcinoma tissues. The expression of miR-1 exhibited the opposite pattern (Fig. 1). FOXC1 is a well-known TF. Knockdown of FOXC1 expression leads to cytoskeleton modification accompanied by a decreased ability of HCC cell proliferation, migration and invasion (32). miR-1 has been reported to be a tumor-suppressor gene that represses cancer cell proliferation and metastasis and promotes apoptosis by ectopic expression (33). The present results support the findings reported in the literature (33). Using technology in which LV3-HOTAIR can promote the expression of the HepG2 gene and tumor growth in animals (Figs. 2 and 3), HOTAIR was observed to promote the migration and invasion of HCC cells by inhibiting RNA binding motif protein 38 (RBM38), which indicates critical roles of HOTAIR and RBM38 in HCC progression (27). In contrast, knockdown of HOTAIR by siRNAs resulted in the reduction of motility and invasion of the human melanoma cell line A375 (34).
IncRNAs regulate gene expression through a variety of mechanisms, including transcription, post-transcriptional processing, chromatin modification, genomic imprinting and regulation of protein function $(35,36)$. Recently, it has been reported that IncRNA transcription can be regulated by key TFs and epigenetic modification (23). For example, p53 can promote IncRNA-p21 transcription, and E2F1 regulates IncRNA E2F1-regulated inhibitor of cell death expression, while the core catalytic subunit of the polycomb repressive complex 2 , enhancer of zeste homolog 2, represses lncRNA sprouty RTK signaling antagonist 4-intronic transcript 1 transcription via epigenetic maintenance of tri-methylation of lysine 27 on histone $\mathrm{H} 3$ (37). c-Myc directly binds to the CCAT1 promoter region and activates IncRNA CCAT1 expression in colon cancer cells (38). In the present study, ChIP assays determined that FOXC1 directly binds to the HOTAIR promoter region. Furthermore, overexpression of FOXC1 increased HOTAIR expression in HCC cells, and knockdown of FOXC1 expression decreased HOTAIR expression in HepG2 cells. FOXC1 also activated lncRNA HOTAIR expression in HCC cells. Taken together, these findings provide clues for exploring the mechanism underlying HOTAIR transcription.

Previous studies have established that IncRNAs can also regulate other non-coding RNAs, in particular miRs, and miRNAs may have an effect on the regulation of IncRNAs $(39,40)$. In support of this notion, it was demonstrated in the current study that HOTAIR-mediated oncogenic activity occurs, at least in part, through the suppression of miR-1. Knockdown of HOTAIR induced the upregulation of miR-1. By contrast, overexpression of miR-1 could downregulate the HOTAIR level, while miR-1 overexpression repressed the HOTAIR level. Thus, HOTAIR and miR-1 may form a reciprocal repression feedback loop. In addition, the mechanism of such a feedback loop was explored in the present study, and it was observed that miR-1 regulates the expression of HOTAIR by directly binding to target sites within the HOTAIR sequence. IncRNA H19 has been demonstrated to inhibit muscle differentiation by antagonizing let-7 (41). It has been reported that long intergenic non-coding RNA muscle differentiation 1 'sponges' miR-133, and that miR-133 regulates muscle differentiation (42). A recently identified lncRNA, cardiac hypertrophy related factor, has been reported to regulate cardiac hypertrophy by targeting miR-489 (43).

It has been recently reported that HOTAIR is a c-Myc-activated driver of malignancy, which acts in part through repressing miR-130a (44). The current study demonstrated that LV3-HOTAIR effectively promotes HepG2 cell formation of clones, as well as growth of animal tumor xenografts. The underlying molecular mechanism may involve the TF FOXC1, which activates the transcription of the lncRNA HOTAIR. The current study suggested another layer of regulation involving ncRNAs (molecular and biological). A better understanding of the ncRNA interaction regulatory network will clearly advance the research in the tumorigenesis of HCC.

\section{Acknowledgements}

The present study was supported by the Natural Science Foundation of Guangdong Province (Guangzhou, China; grant no. 04006966). 


\section{References}

1. Jemal A, Bray F, Center MM, Ferlay J, Ward E and Forman D Global cancer statistics. CA Cancer J Clin 61: 69-90, 2011.

2. Wang P, Ouyang L, Zheng L and Wang Z: Identifying hepatocellular carcinoma-related genes and pathways by system biology analysis. Ir J Med Sci 184: 357-364, 2015.

3. Geisler $\mathrm{S}$ and Coller J: RNA in unexpected places: Long non-coding RNA functions in diverse cellular contexts. Nat Rev Mol Cell Biol 14: 699-712, 2013.

4. Ellis BC, Graham LD and Molloy PL: CRNDE, a long non-coding RNA responsive to insulin/IGF signaling, regulates genes involved in central metabolism. Biochim Biophys Acta 1843: 372-386, 2014

5. Ma L, Bajic VB and Zhang Z: On the classification of long non-coding RNAs. RNA Biol 10: 925-934, 2013.

6. Zhang Z, Zhu Z, Watabe K, Zhang X, Bai C, Xu M, Wu F and Mo YY: Negative regulation of 1ncRNA GAS5 by miR-21. Cell Death Differ 20: 1558-1568, 2013

7. Geng YJ, Xie SL, Li Q, Ma J and Wang GY: Large intervening non-coding RNA HOTAIR is associated with hepatocellular carcinoma progression. J Int Med Res 39: 2119-2128, 2011.

8. Gao JZ, Li J, DU JL and Li XL: Long non-coding RNA HOTAIR is a marker for hepatocellular carcinoma progression and tumor recurrence. Oncol Lett 11: 1791-1798, 2016.

9. Yang Z, Zhou L, Wu LM, Lai MC, Xie HY, Zhang F and Zheng SS: Overexpression of long non-coding RNA HOTAIR predicts tumor recur-rence in hepatocellular carcinoma patients following liver transplantation. Ann Surg Oncol 18: 1243-1250, 2011.

10. Xu ZY, Yu QM, Du YA, Yang LT, Dong RZ, Huang L, Yu PF and Cheng XD: Knockdown of long non-coding RNA HOTAIR suppresses tumor invasion and reverses epithelial-mesenchymal transition in gastric cancer. Int J Biol Sci 9: 587-597, 2013.

11. Kugel JF and Goodrich JA: Non-coding RNAs: Key regulators of mammalian transcription. Trends Biochem Sci 37: 144-151, 2012.

12. Beermann J, Piccoli MT, Viereck J and Thum T: Non-coding RNAs in development and disease: Background, mechanisms, and therapeutic approaches. Physiol Rev 96: 1297-1325, 2016.

13. Mercer TR, Dinger ME and Mattick JS: Long non-coding RNAs: Insights into functions. Nat Rev Genet 10: 155-159, 2009

14. Ma MZ, Chu BF, Zhang Y, Weng MZ, Qin YY, Gong W and Quan ZW: Long non-coding RNA CCAT1 promotes gallbladder cancer development via negative modulation of miRNA-218-5p. Cell death Dis 6: e1583,2015.

15. Meng YX, Niu X and Deng ZH: Inhibitory effect of lentivirus-mediated hTERTp-TK combined with hTERTp-tumstatin on human hepatocarcinoma HepG2 cells. Zhonghua Gan Zang Bing Za Zhi 23: 837-843, 2015 (In Chinese).

16. Braconi C, Kogure T, Valeri N, Huang N, Nuovo G, Costinean S, Negrini M, Miotto E, Croce CM and Patel T: microRNA-29 can regulate expression of the long non-coding RNA gene MEG3 in hepatocellular cancer. Oncogene 30: 4750-4756, 2011.

17. He JH, Li YM, Li YG, Xie XY, Wang L, Chun SY and Cheng WJ: hsa-miR-203 enhances the sensitivity of leukemia cells to arsenic trioxide. Exp Ther Med 5: 1315-1321, 2013.

18. Li D, Feng J, Wu T, Wang Y, Sun Y, Ren J and Liu M: Long intergenic noncoding RNA HOTAIR is overexpressed and regulates PTEN methylation in laryngeal squamous cell carcinoma. Am J Pathol 182: 64-70, 2013.

19. Livak KJ and Schmittgen TD: Analysis of relative gene expression data using real-time quantitative PCR and the $2^{-\Delta \Delta C T}$ method. Methods 25: 402-408, 2001

20. Karreth FA and Pandolfi PP: ceRNA cross-talk in cancer: When ce-bling rivalries goawry. Cancer Discov 3: 1113-1121, 2013.

21. Lipovich L, Johnson R and Lin CY: MacroRNA underdogs in a microRNA world: Evolutionary, regulatory and biomedical significance of mammalian long non-protein-coding RNA. Biochim Biophys Acta 1799: 597-615, 2010.

22. Deng L, Yang SB, Xu FF and Zhang JH: Long noncoding RNA CCAT1 promotes hepatocellular carcinoma progression by functioning as let-7 sponge. J Exp Clin Cancer Res 34: 18, 2015.

23. Lang JH, Li JH, Jiang S, Zhou H and Qu LH: ChIPBase: A database for decoding the transcriptional regulation of long non-coding RNA and microRNA genes fromChIP-Seq data. Nucleic Acids Res 41: D177-D187, 2013.

24. Kornienko AE, Guenzl PM, Barlow DP and Pauler FM: Gene regulation by the act of long non-coding RNA transcription. BMC biology 11: 59, 2013
25. Rinn JL, Kertesz M, Wang JK, Squazzo SL, Xu X, Brugmann SA, Goodnough LH, Helms JA, Farnham PJ, Segal E and Chang HY: Functional demarcation of active and silent chromatin domains in human HOX loci by noncoding RNAs. Cell 129: 1311-1323, 2007.

26. Huang L, Liao LM, Liu AW, Wu JB, Cheng XL, Lin JX and Zheng M: Overexpression of long noncoding RNA HOTAIR predicts a poor prognosis in patients with cervical cancer. Arch Gynecol Obstet 290: 717-723, 2014.

27. Ding C, Cheng S, Yang Z, Lv Z, Xiao H, Du C, Peng C, Xie H, Zhou L, Wu J and Zheng S: Long non-coding RNA HOTAIR promotes cell migration and invasion via down-regulation of RNA binding motif protein 38 in hepatocellular carcinoma cells. Int J Mol Sci 15: 4060-4076, 2014.

28. Kim K, Jutooru I, Chadalapaka G, Johnson G, Frank J, Burghardt R, Kim S and Safe S: HOTAIR is a negative prognostic factor and exhibits pro-oncogenic activity in pancreatic cancer. Oncogene 32: 1616-1625, 2013.

29. Fu WM, Zhu X, Wang WM, Lu YF, Hu BG, Wang H, Liang WC, Wang SS, Ko CH, Waye MM, et al: Hotair mediates hepatocarcinogenesis through suppressing miRNA-218 expression and activating P14 and P16 signaling. J Hepatol 63: 886-895, 2015.

30. Li C, Chen J, Zhang K, Feng B, Wang R and Chen L: Progress and prospects of long noncoding RNAs (lncRNAs) in hepatocellular carcinoma. Cell Physiol Biochem 36: 423-434, 2015.

31. Bhan A, Hussain I, Ansari KI, Kasiri S, Bashyal A and Mandal SS: Antisense transcript long noncoding RNA (lncRNA) HOTAIR is transcriptionally induced by estradiol. J Mol Biol 425: 3707-3722, 2013.

32. Xu ZY, Ding SM, Zhou L, Xie HY, Chen KJ, Zhang W, Xing CY, Guo HJ and Zheng SS: FOXC1 contributes to microvascular invasion in primary hepatocellular carcinoma via regulating epithelial-mesenchymal transition. Int J Biol Sci 8: 1130-1141, 2012.

33. Han C, Yu Z, Duan Z and Kan Q: Role of MicroRNA-1 in human cancer and its therapeutic potentials. Biomed Res Int 2014: 428371, 2014.

34. Tang L, Zhang W, Su B and Yu B: Long noncoding RNA HOTAIR is associated with motility, invasion and metastatic potential of metastatic melanoma. Biomed Res Int 2013: 251098, 2013.

35. Gibb EA, Brown CJ and Lam WL: The functional role of long non-coding RNA in human carcinomas. Mol Cancer 10: 38, 2011.

36. Bernard D, Prasanth KV, Tripathi V, Colasse S, Nakamura T, Xuan Z, Zhang MQ, Sedel F, Jourdren L, Coulpier F, et al: A long nuclear-retained non-coding RNA regulates synaptogenesis by modulating gene expression. EMBO J 29: 3082-3093, 2010.

37. Liu Q, Huang J, Zhou N, Zhang Z, Zhang A, Lu Z, Wu F and Mo YY: LncRNA loc285194 is a p53-regulated tumor suppressor. Nuclei Acids Res 41: 4976-4987, 2013

38. Yang F, Xue X, Bi J, Zheng L, Zhi K, Gu Y and Fang G: Long noncoding RNA CCAT1, which could be activated by c-Myc, promotes the progression of gastric carcinoma. J Cancer Res Clin Oncol 139: 437-445, 2013.

39. Mattick JS and Makunin IV: Non-coding RNA. Hum Mol Genet 15: R17-R29, 2006

40. Gibb EA, Brown CJ and Lam WL: The functional role of long non-coding RNA in human carcinomas. Mol Cancer 10: 38-55, 2011.

41. Kallen AN, Zhou XB, Xu J, Qiao C, Ma J, Yan L, Lu L, Liu C, Yi JS, Zhang $\mathrm{H}$, et al: The imprinted H19 lncRNA antagonizes let-7 microRNAs. Molecular cell 52: 101-112, 2013.

42. Legnini I, Morlando M, Mangiavacchi A, Fatica A and Bozzoni I: A feedforward regulatory loop between $\mathrm{HuR}$ and the long noncoding RNA linc-MD1 controls early phases of myogenesis. Mol Cell 53: 506-514, 2014

43. Wang K, Liu F, Zhou LY, Long B, Yuan SM, Wang Y, Liu CY, Sun T, Zhang XJ and Li PF: The long noncoding RNA CHRF regulates cardiac hypertrophy by targeting miR-489. Circ Res 114: 1377-1388, 2014

44. Ma MZ, Li CX, Zhang Y, Weng MZ, Zhang MD, Qin YY, Gong W and Quan ZW: Long non-coding RNA HOTAIR, a c-Myc activated driver of malignancy, negatively regulates miRNA-130a in gallbladder cancer. Mol Cancer 13: 156, 2014. 